\title{
EDUCAÇÃO PARA A WEB SEMÂNTICA: a contribuição dos cursos on-line abertos e massivos
}

\author{
Vitor Gonçalves \\ CIEB, Instituto Politécnico de Bragança, Portugal \\ vg@ipb.pt \\ José António Moreira \\ Universidade Aberta, Portugal \\ jmoreira@uab.pt
}

\section{RESUMO}

A web é o maior repositório de informação que disponibiliza conteúdos multimédia. Contudo, a sua localização não é uma tarefa fácil, principalmente, porque sua semântica ou significado só podem ser capturados em seu contexto e de acordo com a perspetiva humana. Nos últimos anos, o World Wide Web Consortium e a comunidade científica internacional têm vindo a realizar esforços significativos, com o objetivo de melhorar a localização, recuperação e reutilização de informações, que podem estar inacessíveis ou armazenadas em servidores espalhados pelo mundo na deep web ou na web semântica. Metadados e ontologias, ferramentas de anotação, sistemas de agentes, entre outros, são os principais elementos para o desenvolvimento de soluções que levam à mudança da realidade da web. Tendo em consideração este contexto, e através de uma análise exploratória, o objetivo desta pesquisa é identificar os MOOCs existentes orientados para o estudo da web semântica e analisar o tipo de conteúdo abordado.

Palavras-chave: Web Semântica. MOOC. Formação On-line.

\section{EDUCATION FOR THE SEMANTIC WEB: the contribution of the massive open online courses}

\begin{abstract}
The web is a largest information repository which makes multimedia contents available. However, their localization is not an easy task, mainly since their semantics or meaning can only be captured in their context and in accordance to human perspective. In the past years, the World Wide Web Consortium and the international scientific community have been carrying out significant efforts with view to improving the localization, retrieval and reuse of information, which may be inaccessible or stored in servers scattered around the deep web or the semantic web. Metadata and ontologies, annotation tools, ontologies tools, agent systems, among others, are the key elements for the development of solutions that are gradually lead to changing the web reality. In this context, we intend, essentially, through an
\end{abstract}


exploratory research, to identify MOOCs oriented towards the study of the semantic web and, consequently, determine the typology of the content covered.

Keywords: Semantic web. MOOC. Online Training

\section{EDUCACIÓN PARA LA WEB SEMÁNTICA: la contribución de cursos en línea abiertos y masivos}

\section{RESUMEN}

La web es el mayor depósito de información que proporciona contenido multimedia. Sin embargo, su localización no es una tarea fácil, principalmente porque su semántica o significado solo se puede capturar en su contexto y de acuerdo con la perspectiva humana. En los últimos años, el Consorcio de la World Wide Web y la comunidad científica internacional han realizado importantes esfuerzos para mejorar la localización, la recuperación y la reutilización de la información, que puede ser inaccesible o almacenada en servidores de todo el mundo en la web profunda o en web semántica. Los metadatos y las ontologías, las herramientas de anotación, los sistemas de agentes, entre otros, son los elementos principales para el desarrollo de soluciones que conducen a cambiar la realidad de la web. Teniendo en cuenta este contexto, y através de un análisis exploratorio, el objetivo de esta investigación es identificar los MOOC existentes orientados al estudio de la web semántica y analizar el tipo de contenido abordado.

Palabras clave: Web semántica. MOOC. Formación en línea. 


\section{INTRODUÇÃO}

A afirmação da adoção e utilização de tecnologias digitais em diversos setores da sociedade, incluindo o educativo, tem vindo a assumir particular importância e a caracterizar a investigação nas últimas décadas. Muitos modelos e projetos de integração das tecnologias digitais em contexto educativo têm contribuído para facilitar a concretização de diversos processos de aprendizagem formais e informais através da simplificação de um conjunto de tarefas inerentes a esses processos.

A web semântica pode ser vista como uma das últimas eras da web com vista a gerar uma web mais inteligente. Mas poderá a web semântica afirmar-se como um espaço educativo com uma pedagogia própria? Quais as mudanças que provoca(rá) na educação e onde se pode aprender ou obter mais (in)formação sobre esta temática?

A web semântica pode, na realidade, contribuir para uma web mais inteligente na medida em que atribui significado ao conteúdo dos documentos da web facilitando assim a sua interpretação pelos humanos e pelos não humanos.

Com o objetivo de responder a estas questões, propomo-nos neste texto identificar os Massive Open Online Courses (MOOC) existentes na web orientados para a temática da web semântica. A opção pelos MOOC para suportar e promover cursos sobre esta temática, relaciona-se com o facto desta modalidade ser relativamente recente e poder fomentar "uma nova gama de possibilidades desafiantes para ampliar o acesso a uma educação de qualidade" (TEIXEIRA et al., 2015, p. 9). Para esta análise iremos realizar um estudo exploratório, utilizando como fontes principais de informação artigos e publicações nesta área.

Assim, com esta pesquisa pretende-se recolher informação que permita o desencadear de um debate mais alargado sobre a web semântica e o papel que pode assumir na criação de processos pedagógicos diferenciados mediados e enriquecidos com tecnologias digitais.

\section{A WEB SEMÂNTICA}

A web semântica é uma iniciativa, liderada pelo W3C, que pretende criar um meio universal para partilha de informação, construindo uma web de dados ligados e atribuindo significado (semântica) ao conteúdo dos documentos da web, de modo a que esse significado seja compreendido pelos humanos e não humanos. A web semântica corresponde a uma extensão da web atual que permite encontrar, partilhar e (re)combinar informação mais facilmente.

A missão do projeto web semântica pode traduzir-se na condução da web rumo ao seu verdadeiro potencial (MILLER, 2004). Os seus principais objetivos prendem-se: construir uma estrutura comum de representação dos dados de modo a facilitar a integração de diversas fontes de informação para extrair novo conhecimento (só é possível através da 
migração de uma web de documentos para uma web de dados); aumentar a utilidade da informação conectando-a aos seus conceitos e ao seu contexto; e, descobrir e analisar, de forma mais eficiente, a informação (MILLER, 2004).

Através da web semântica é possível retirar mais proveito das hiperligações, uma vez que os links passam a fornecer as relações entre os recursos de informação, permitindo que as máquinas tenham acesso a mais camadas de informação e, consequentemente, possam trabalhar e partilhar conhecimento com os humanos.

A Quadro 1 resume as mudanças da web atual ou convencional para a web semântica.

Quadro 1 - Caracterização da web.

\begin{tabular}{|c|c|c|}
\hline Característica & Web convencional & Web semântica \\
\hline Destinatários & Seres humanos & $\begin{array}{l}\text { Seres humanos e computadores } \\
\text { (agentes de software) }\end{array}$ \\
\hline Linguagem principal & $\begin{array}{l}\text { HTML (HyperText } \\
\text { Markup Language) }\end{array}$ & XML (eXtensible Markup Language) \\
\hline Estrutura dos conteúdos & $\begin{array}{l}\text { Documentos não } \\
\text { estruturados }\end{array}$ & Documentos estruturados \\
\hline Exibição dos conteúdos & $\begin{array}{l}\text { Dados e sua } \\
\text { apresentação no } \\
\text { mesmo documento }\end{array}$ & $\begin{array}{l}\text { Dados correspondem a um } \\
\text { documento XML e apresentação } \\
\text { corresponde a documentos XSL de } \\
\text { acordo com as interfaces }\end{array}$ \\
\hline Edição dos conteúdos & Documentos estáticos & Documentos dinâmicos \\
\hline Interatividade & Difusão unidirectional & Difusão bidirecional (web editável) \\
\hline Semântica dos conteúdos & Semântica implícita & $\begin{array}{l}\text { Semântica explicita (metadados e } \\
\text { ontologias) }\end{array}$ \\
\hline $\begin{array}{l}\text { Decomposição e } \\
\text { recomposição }\end{array}$ & $\begin{array}{l}\text { websites } \\
\text { independents }\end{array}$ & $\begin{array}{l}\text { Agregação num website dinâmico } \\
\text { de conteúdos de diversos websites }\end{array}$ \\
\hline Publicação & $\begin{array}{l}\text { Centralizada (Servidor } \\
\text { Web) }\end{array}$ & Descentralizada \\
\hline
\end{tabular}

A estruturação da informação para a web semântica parece assentar no uso intensivo de metadados, o que permitirá o acesso automático à informação por parte das máquinas, através do processamento semântico dos dados e de heurísticas (BERNERSLEE et al., 2001).

Neste sentido, no decorrer da primeira década do milénio, começaram a surgir algumas arquiteturas para a compreensão mais alargada da web semântica. Arquiteturas baseadas em camadas sobrepostas, onde cada camada deveria ser complementar com as camadas inferiores, mas sem qualquer dependência da camada superior, sendo que em 2002, foram apresentadas algumas mudanças que permitiram clarificar a arquitetura para a web semântica (BERNERS-LEE, 2002). Mais tarde, em 2005, e face às dificuldades de integração e de compatibilidade das camadas, surgiram novas alterações.

Neste sentido, conforme evidenciado na Figura 1, apresenta-se o modelo mais recente da arquitetura para a web semântica que se pode dividir em quatro camadas principais que identificam as funções e as características fundamentais das tecnologias: camada estrutural, sintática, semântica e lógica. 
A camada estrutural permite estruturar os dados, identificar os recursos de forma única e fornecer os meios para a representação, armazenamento e intercâmbio de informação, garantindo, deste modo, a integridade e confidencialidade dos dados. A camada sintática possibilita a estruturação dos dados associados a um recurso e à verificação da consistência desse recurso, de acordo com regras sintáticas formalmente descritas e validadas. A camada semântica é responsável pela criação de vocabulários para a descrição semântica dos recursos e para a definição das relações existentes entre estes. A camada lógica permite declarar regras lógicas que podem ser executadas pelos agentes de software para realizar inferências automáticas e verificar o nível de coerência lógica dos recursos.

Figura 1 - Arquitetura para a web semântica.

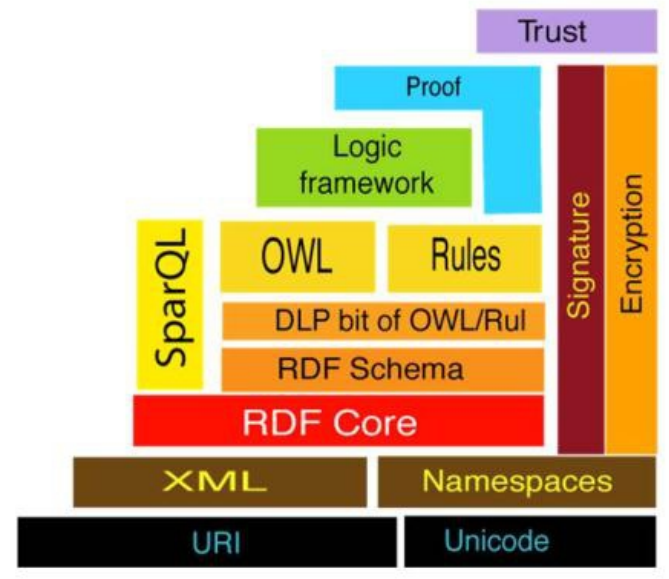

Fonte: Berners-Lee (2005).

A evolução da web para a web semântica, descrita por Berners-Lee, Hendler e Lassila em 2001, tem demorado a concretizar-se. Contudo, atualmente, milhões de domínios web contêm aplicações ou simplesmente marcações nas suas páginas web dando um passo silencioso rumo à web semântica.

\section{MASSIVE OPEN ONLINE COURSES (MOOC)}

Os Massive Open Online Courses (MOOC) tiveram como antecessores os Recursos Educativos Abertos (REA) e a Aprendizagem Social Aberta (ASA): "a origem dos MOOC parece estar ligada a dois fenómenos: Open Educational Resources (OER) e Open Social Learning (OSL)" (MONTERREY, 2014, p. 3). O conceito de OLS surge "de forma espontânea como uma nova forma de entender o ensino e a aprendizagem, com base na descoberta de práticas facilitadas por certas ferramentas digitais" (UNESCO, 2012, p. 7) e pode ser entendido "como uma atividade de aprendizagem ativa, autogestionada, de natureza não formal ou informal, que se realiza através do uso das ferramentas tecnológicas da web social ou da web 2.0" (DOMíNGUEZ, 2011, p. 4). O termo OER surgiu pela primeira 
vez numa conferência apoiada pela UNESCO em 2002, e nessa altura foram definidos como "um conjunto aberto de recursos educativos potenciados pelas tecnologias de informação e comunicação, servindo para consulta, uso e adaptação e novamente reutilizados por uma comunidade de utilizadores com propósitos não comerciais" (JOHNSTONE, 2005, p. 16). Interessa salientar que, após uma década, a UNESCO (2012) atualiza o conceito, considerando os OER como:

Materiais de ensino, aprendizagem e investigação em quaisquer suportes, digitais ou outros, que se situem no domínio público ou que tenham sido divulgados sob licença aberta que permite acesso, uso, adaptação e redistribuição gratuitos por terceiros, mediante nenhuma restrição ou poucas restrições.( UNESCO,2012,p.1).

Os OER permitiram aumentar o acesso democrático ao conhecimento e a racionalização de despesas, promovendo uma nova ecologia do conhecimento que, consequentemente, impulsionou o conectivismo proposto por George Siemens e Stephen Downes (LITTO, 2006). O conectivismo, teoria de aprendizagem que suporta os MOOC, surge para suprir as modificações económicas, culturais e sociais que o desenvolvimento tecnológico acarreta e "fornece uma perceção das habilidades e tarefas de aprendizagem necessárias para os aprendizes florescerem na era digital” (SIEMENS, 2005, p. 8). O conectivismo que entende a aprendizagem como um processo que ocorre dentro de ambientes nebulosos onde os elementos centrais estão em mudança, não inteiramente sob o controle das pessoas" (SIEMENS, 2005, p. 5).

Ainda de acordo com Siemens (2013), os MOOC assumem-se como "uma continuação da tendência em inovação, experimentação e do uso da tecnologia iniciada pelo ensino a distância e on-line, para oferecer oportunidades de aprendizagem de forma massiva" (2013, p. 5). Os MOOCs fornecem acesso aberto, baseado num modelo de educação a distância, promovendo uma participação interativa em larga escala (MA DLEE et al., 2013) e "integram redes sociais, recursos on-line acessíveis e são facilitados por profissionais líderes no campo de estudo" (MCAULEY et al., 2010, p. 4), ou seja:

Representam uma inovação e a incorporação e o uso da tecnologia como meio para difundir o que antes estava restrito aos muros das instituições. Foi pensado para atingir milhões, bastando para isto uma conexão de internet, a disponibilidade e a vontade do usuário. (ALMEIDA; MANOLE, 2013, p. 25).

No que respeita à classificação dos MOOCs, apesar da diversidade de tipologias e variantes, parece existir convergência na literatura ao aceitar a distinção dos MOOCs proposta por Downes (2012), que designa uns por cMOOC e os outros por xMOOC (WATTERS, 2012). Apesar das duas tipologias terem características comuns como, por exemplo, os conteúdos digitais divididos em semanas, as principais diferenças resultam do papel dos professores e dos participantes no MOOC e da forma como a aprendizagem 
ocorre.

Os cMOOC "são estruturados a partir da aprendizagem auto-organizada, centrada em obter significado através da experiência em comunidade, utilizando ferramentas de participação como blogs, feeds RSS e outros métodos descentralizados" (TORRES, 2013, p. 66). São centrados nos contextos, correspondem a uma perspetiva conectivista e "colocam ênfase na criação de conhecimento pelos alunos, criatividade, autonomia e aprendizagem social e colaborativa" (MONTERREY, 2014, p. 4). O Connectivism and Connective Knowledge Course (CCK08), o Personal Learning Environments Networks and Knowledge (PLENK2010) e o Learning Analytics and Knowledge (LAK12) são alguns dos exemplos de cursos desta tipologia.

Os XMOOC seguem um projeto de curso instrucionista no qual os objetivos de aprendizagem são predefinidos pelo instrutor (LITTLEJOHN, 2013) e os materiais são projetados e preparados com antecedência. Os xMOOC são originários do MIT/Stanford, centram-se nos conteúdos e possuem uma organização mais rígida, que limita a criatividade: $\mathrm{O}$ "x" corresponde à modalidade de MOOC normalmente mais comercial, ou seja, aqueles que são oferecidos através de plataformas comerciais ou semi-comerciais, como a Coursera, edX e Udacity (MONTERREY, 2014). Neste género de cursos, os alunos assistem a séries de vídeo (palestras), fazem a leitura de artigos recomendados e resolvem questionários (BALI, 2014). Além destas duas tipologias (cMOOC e xMOOC), posteriormente surgiram diversas propostas especificando a tipologia de utilização, no entanto tem sido da responsabilidade de cada utilizador ou promotor, optar pelo modelo mais adequado de acordo com os objetivos específicos de aprendizagem.

Assim, torna-se fundamental que os promotores e utilizadores possuam as competências tecnológicas necessárias para operar neste novo universo tecnológico, onde as tecnologias assumem um papel preponderante, já que permitirão tirar partido dos mais recentes ambientes de aprendizagem que distribuem MOOC.

Finalmente, ainda no que respeita aos MOOC, importa salientar que este género de cursos parece surgir como uma oportunidade de inovação tecnológica, pois "do ponto de vista das IES [Instituições de Ensino Superior], os MOOC servem para a divulgação das universidades, favorecendo o recrutamento de novos estudantes e também como campo para experimentos pedagógicos" (RIEDO et al., 2014, p. 5).

É esse o caso da para a iniciativa Aula Aberta (https://aulaberta.uab.pt/), promovida pela Universidade Aberta de Portugal, que desde 2017, se assume como um espaço de partilha com a comunidade, disponibilizando em acesso aberto um conjunto de temas e de recursos, em áreas que são trabalhadas em cursos formais oferecidos pela Universidade. Através da Aula Aberta possibilita-se a um público alargado um primeiro contacto, ou uma nova experiência, com o contexto da aprendizagem on-line.

O participante na iniciativa Aula Aberta encontra percursos breves de aprendizagem que introduzem temas atuais e possibilitam momentos de partilha de ideias e de experiências, nomeadamente através das redes sociais associadas aos cursos. 0 participante pode, ainda, navegar livremente entre os vários temas apresentados, explorar 
os recursos multimédia e participar nas atividades propostas. Os percursos da formação disponibilizada estruturam-se como espaços de aprendizagem abertos, sendo que alguns integram-se no formato MOOC.

\section{METODOLOGIA}

O presente estudo concretiza-se através de uma pesquisa exploratória documental, utilizando como fontes principais de informação publicações no âmbito da identificação de MOOC orientados para o estudo e compreensão da temática da web semântica. Assim, e como já foi destacado no ponto introdutório, pretendemos identificar quais os MOOCs orientados para o estudo da web semântica (primeiramente em língua portuguesa e posteriormente espanhol, francês ou inglês) e quais os conteúdos abordados nesses cursos.

Para uma melhor organização e categorização dos dados provenientes da análise exploratória dos cursos da web semântica identificados estabelecemos o seguinte conjunto de categorias de análise: plataforma MOOC, designação do curso, promotor, duração do curso, idioma, conteúdos abordados, recursos utilizados, certificação, creditação, entre outros critérios relevantes.

A recolha de dados foi concretizada, no final do ano de 2019, entre os dias 01 de outubro a 30 de novembro, por meio de uma pesquisa exploratória, com base nos parâmetros referidos anteriormente. Interessa referir que foram ignorados cursos que apenas se baseavam no estudo de uma determinada tecnologia semântica. Para além disso, optou-se por efetuar uma pesquisa apenas nas plataformas MOOCs mais conhecidas e utilizadas em todo o mundo: Coursera, EdX, Udacity, Udemy, Iversity, Khan Academy, FutureLearn, OpenUpEd e Míriada X. Os resultados corresponderam aos disponíveis no agregador deste tipo de cursos Mooc List (https://www.mooc-list.com), incluindo cursos de plataformas instaladas por universidades, tais como OpenHPI, Open edX ou Fun MOOC. Apesar do baixo número de cursos disponíveis nestas plataformas, evitou-se alargar o espetro a instituições de ensino superior que disponibilizam a temática da web semântica em formato MOOC.

Após recolhidos os dados, foi efetuada uma análise de conteúdo para, deste modo, se identificarem os conteúdos abordados sobre a temática da web semântica e,

\section{APRESENTAÇÃO E DISCUSSÃO DOS RESULTADOS}

Através do desenvolvimento da presente pesquisa, identificamos os MOOCs relacionados ao tema da web semântica. Usando os termos "Semantic Web" em listas específicas (https://www.mooc-list.com/tags/semantic-web) e mecanismos de busca das principais plataformas de MOOC, constatou-se que a oferta deste tipo de curso é francamente escassa, considerando a importância que a rede semântica assume na 
sociedade atual. Todos eles abordaram os principais conceitos e tecnologias da web semântica. No entanto, exceto um deles -Web of Data- que apresentava exercícios de aplicação, todos correspondem a cursos para obter conhecimentos essenciais sobre a web semântica e suas tecnologias, e não a cursos que permitem aprender a criar aplicativos ou conteúdo com essas tecnologias para esta nova geração da web. Realizada a pesquisa no agregador de $\mathrm{MOOC}$ e nas plataformas MOOCs referidas, foram identificados catorze cursos: "Web of Data" (Coursera); "La Web Semántica: Herramientas para la publicación y extracción efectiva de información en la Web" (Coursera); "Content Writing in the Semantic Web" (Udemy), "Learn Semantic Web - Next Phase of WWW in 1 Hour" (Udemy); "Semantic Web in 1 hour" (Udemy); Dynamics of Knowledge Organisation (Udemy); "Introduction to Linked Data and the Semantic Web" (FutureLearn); "Semantic Web and Linked Data" (Míriada X); "La Web 3.0 en la Educación" (Míriade X); "Introduction to a Web of Linked Data" (FUN); "Semantic Web Technologies" (OpenHPI); "Knowledge Engineering with Semantic Web Technologies" (openHPI); "Web Sémantique et Web de Données" (Fun MOOC); "Introduction to a Web of Linked Data" (Fun MOOC). De referir que foram apenas pesquisados MOOC nessas plataformas, usando as palavras-chave "Semantic web" nas línguas portuguesa, inglesa, espanhola e francesa. Genericamente, os resultados apontam para cursos onde são abordados os seguintes conteúdos: Knowledge and the Web of Data; $X M L$ and RDF; Ontologies and Logic; OWL, Rules, and Reasoning. A tipologia destes MOOC corresponde maioritariamente a XMOOC (Self-paced ou similar). Os conteúdos disponibilizados são essencialmente vídeos, textos e testes que abordam os principais conceitos e tecnologias da web semântica. A certificação é possível desde que seja realizado o seu pagamento.

\section{CONSIDERAÇÕES FINAIS}

Através do desenvolvimento da presente pesquisa, identificámos os MOOCs relacionados com o tema da web semântica. Essa identificação, quer dos cursos, quer dos seus conteúdos, permitiram-nos concluir que existe, ainda, um número muito reduzido de cursos nesta área.

Será que a web precisará evoluir mais para que se generalize a criação de conteúdos para a web semântica? Provavelmente sim, mas isso não justifica o facto de não haver mais cursos em face da nova realidade semântica emergente.

Com efeito, verificámos que a oferta deste tipo de cursos é francamente escassa face à importância que a web semântica assume na sociedade atual. Verificámos, ainda, que todos os cursos identificados abordam os principais conceitos e tecnologias da web semântica, no entanto não analisam a forma como se pode aprender a realizar aplicações ou conteúdos para esta nova geração da web. Torna-se, pois, necessário que os promotores dos MOOCs, especialmente as instituições de ensino superior, invistam em cursos sobre essa temática, principalmente no que se refere ao contributo que esta tipologia de cursos 
poderá oferecer no desenvolvimento de aplicações que nos permitam caminhar no sentido de uma web mais inteligente.

Para além disso, será importante também aprofundar a temática em análise, porque, e apesar de não termos uma resposta concreta a esta questão, cremos que a web semântica pode assumir um papel relevante na criação de Ambientes Virtuais de Aprendizagem (AVA), onde possam ser desenvolvidas práticas pedagógicas diferenciadas e inclusivas, enriquecidas e mediadas pelas tecnologias digitais.

\section{REFERÊNCIAS}

ALMEIDA, F.; MANOLE, D. Aula essencial e suas expansões em cursos massivos, abertos e online de aprendizagem (MOOC): conceitos, experiências, desafios e perspectivas no Brasil. XI Encontro de Pesquisadores do Programa de Pós-Graduação em Educação e Currículo, PUC-SP, 2013, p. 2-13. Disponível em: http://www.ced.pucsp.br/encontro_pesquisadores_2013/downloads/anais_encontro_2013/oral/fern ando_jose_almeida_daniela_manole.pdf. Acesso em: 15 jan. 2020.

BALI, M. MOOC pedagogy: gleaning good practice from existing MOOCs. Journal of Online Learning and Teaching, v.10, n.1, p. 44-56, 2014.

BERNERS-LEE. The Semantic Web. Center for eBusiness@MIT, 2002.

BERNERS-LEE. Semantic Web Concepts. WWW Consortium, MIT Computer Science \& Artificial Inteligence Laboratory, 2005. Disponível em: http://www.w3.org/2005/Talks/0517-boittbl/. Acesso em: 15 jan. 2020.

BERNERS-LEE, T.; HENDLER, J.; LASSILA, O. The Semantic Web: A new form of web content that is meaningful to computers will unleash a revolution of new possibilities [Electronic resource]. Scientific American, 2001.

DOMÍNGUEZ, D. El Open \& Social Learning y su potencial de transformación socioeducativa. Intervención Sociocomunitaria. Madrid: UNED, 2011.

DOWNES, S. Massively Open Online Courses Are "Here to Stay", 2012.

JOHNSTONE, S. M. Open educational resources serve the world. Educause Quarterly, v. 28, n. 3, 15-26, 2005.

LITTLEJOHN, A. Understanding massive open online courses. CEMCA EdTech Notes, 2013.

LITTO, F. M. A nova ecologia do conhecimento: conteúdo aberto, aprendizagem e desenvolvimento. Inclusão Social, v. 1, n. 2, p. 15-26, 2006.

MA DLEE, KELLY, CKUO, GRACE., J. A massive open online course on pharmacogenomics: not just disruptive innovation but a possible solution. Pharmacogenomics, v. 14, n. 10, p. 1125-1127, 2013.

MCAULEY, A., STEWART, B., SIEMENS, G., \& CORMIER, D. The MOOC model for digital practice. SSHRC Knowledge Synthesis Grant on the Digital Economy, 2010. Disponível em: http://www.edukwest.com. Acesso em: 15 jan. 2020.

MILLER, E. Weaving meaning: An overview of the Semantic Web. University of Michigan: Michigan USA, 2004. 
MONTERREY, T. Edu Trends Report, 2014. Disponível em:

http://observatory.itesm.mx/edutrendsmooc/. Acesso em: 15 jan. 2020.

RIEDO, C. R. F.; AGUIAR PEREIRA, E. M.; WASSEM, J.; GARCIA, M. F. O desenvolvimento de um MOOC (Massive Open Online Course) de Educação Geral voltado para a formação continuada de professores: Uma breve análise de aspectos tecnológicos, econômicos, sociais e pedagógicos. SIED: EnPED-Simpósio Internacional de Educação a Distância e Encontro de Pesquisadores em Educação a Distância, 2014, p. 70-78.

SIEMENS, G. Connectivism: a theory of learning for the digital age. International Journal of Instructional Technology and Distance Learning, v. 2, n. 1, p. 17-29, 2005.

SIEMENS, G. Massive open online courses: Innovation in education. Open Educational Resources: Innovation, Research and Practice, 2013.

TEIXEIRA, A.; MOTA, J.; MORGADO, L.; SPILKER, M. iMOOC: Um Modelo Pedagógico Institucional para Cursos Abertos Massivos Online (MOOCs). Educação, Formação \& Tecnologias, v. 8, n. 1, p. 4-12, 2015.

TORRES, D. Reflexiones y primeiros resultados de MOOCs em Iberoamerica: UNEDCOMA y UNX. Revista Científica Iberoamericana de Tecnologia Educativa, v. 2, n.1, p. 15-26, 2013.

UNESCO. Congresso Mundial Sobre Recursos Educacionais Abertos (REA). Paris, 2012. WATTERS, A. Top Ed-Tech Trends of 2012: MOOCs. Hack Education, 2012.

\section{BIOGRAFIA DOS AUTORES}

VITOR GONÇALVES- Doutor em Engenharia Eletrotécnica e dos Computadores e Mestre em Tecnologia Multimédia. Professor-adjunto no Departamento de Tecnologia Educativa e Gestão de Informação do Instituto Politécnico de Bragança. Para além da lecionação e orientação de estágios e dissertações, o seu trabalho incidiu também na publicação de 5 capítulos em livros e mais de 35 artigos em publicações de revistas ou artigos de conferências e na participação de projetos nacionais (Portal dos Catraios, Ciência Bragança e INTEGRA(-TE) e internacionais (INTACT e AduLeT).

JOSÉ ANTÓNIO MOREIRA - Doutor e Mestre em Ciências da Educação pela Universidade de Coimbra. Realizou um Pós-Doutoramento em Tecnologias Educacionais e da Comunicação também pela Universidade de Coimbra. Licenciatura em História da Arte pela Faculdade de Letras da Universidade de Coimbra. É Professor no Departamento de Educação e Ensino a Distância da Universidade Aberta, Portugal. Atualmente é Diretor da Delegação Regional do Porto da Universidade Aberta e Coordenador da Unidade de Desenvolvimento dos Centros Locais de Aprendizagem da mesma universidade. Investigador e Coordenador do Núcleo de Estudos de Pedagogia no Ensino Superior sedeado no Centro de Estudos Interdisciplinares do Século XX. 